\title{
Monitoring of Multimode Processes Based on Quality-Related Common Subspace Separation
}

\author{
Yunpeng Fan, Shipeng Li, and Yingwei Zhang \\ State Laboratory of Synthesis Automation of Process Industry, Northeastern University, Shenyang, Liaoning 110819, China \\ Correspondence should be addressed to Yingwei Zhang; zhangyingwei@mail.neu.edu.cn
}

Received 5 December 2013; Accepted 16 March 2014; Published 13 April 2014

Academic Editor: Lixian Zhang

Copyright (c) 2014 Yunpeng Fan et al. This is an open access article distributed under the Creative Commons Attribution License, which permits unrestricted use, distribution, and reproduction in any medium, provided the original work is properly cited.

\begin{abstract}
A new monitoring approach for multimode processes based on quality-related common subspace separation is proposed. In the model, the data set forms a larger space when the correlation between process variables and quality variables is considered. And then the whole space is decomposed: quality-related common subspace, quality-related specific subspace, and the residual subspace. Monitoring method is performed in every subspace, respectively. The simulation results show the proposed method is effective.
\end{abstract}

\section{Introduction}

Multimode is one of the characteristics of the networked control systems. Adding product quality information into data set for complex process monitoring is a good approach in industrial process. It will be easier to monitor and forecast the product quality online if these data are utilized reasonably in process monitoring and fault diagnosis field. As we all know, in many production processes, the quality indexes of products are obtained by experimental analysis offline instead of being measured directly online owing to the limitations of existing technology [1-6]. However, data can be obtained online in some processes of chemical industry field, where we can get a mass of data directly and timely, and the quality information can also be contained there. To find the internal relations of the obtained data, thousands of domestic and foreign scholars have made a great effort, and many effective methods have been proposed to realize the quality monitoring and forecasting of products online [7].

Traditional quality monitoring and forecasting methods based on analytical model are highly dependent on the precise mathematical model of the object being diagnosed; thus its practical application is limited. Since multivariate statistical regression methods such as principal component regression (PCR) $[8,9]$ and partial least squares (PLS) [10] have overcome the deficiencies described above in recent years, they are becoming the hotspot in quality monitoring and online forecasting field that the contemporary scholars at home and abroad focus on [11-16].

As a powerful multivariate statistical method, PLS can not only establish the relationship between quality data and process data accurately but also maintain good robustness with the assumption that the data obey a Gaussian distribution. It is an effective tool for monitoring the multivariable industrial process, which can be described by linear model. In the case of nonlinear data, traditional PLS may have a great error or even fail to establish the regression model. Despite all this, the PLS method can still be used to solve this problem if we do more preliminary work. Kim and Rosipal mapped the nonlinear data from the original space into a high-dimensional space, and they found the approximate linearity between these data in the new space and then utilized PLS. In this way, the regression problem for nonlinear data was resolved. In general, we call this preliminary work a kernel trick; the modified PLS is named KPLS which is short for kernel partial least squares. KPLS can also reduce the dimension of the sample space and eliminate the noise and correlation of data.

To solve the monitoring problems for multimode processes, various strategies have been proposed and put into process monitoring $[17,18]$. Sub-PLS modeling algorithm has been developed to solve the multimode problem $[19,20]$. In general, the monitoring programs will be adjusted according 
to the different modes; that is, the monitoring program will be switched when the mode switches from one to another. Wang et al. studied the monitoring approach based on mode identification for multimode process with transitions [21]. However, the monitoring system often malfunctions in the transition processes. In this paper, considering that in the same system the common part exists in most modes, monitoring the common part and specific part, respectively, is a good way to reduce fault rate.

In this paper, a multimode process monitoring method based on quality-related common subspace separation is proposed. On the basis of the common subspace separation method, we consider the correlation between process variables and quality variables in model using KPLS, and then we separate the whole space into three parts: quality-related common subspace, quality-related specific subspace, and the residual subspace. At last, we monitor the fault in every subspace, respectively. Experiment results show the proposed method is effective. The advantages of this paper's methods are as follows. (1) The characteristic relationships between different modes are considered, and the complexity and fault rate of the monitoring program are reduced. (2) Fault can be detected accurately and timely in one of the three subspaces when it occurs. (3) Quality-related common subspace reflects local structural information of input data variables in each mode and has the strong ability to explain quality variables.

\section{Quality-Related Common Subspace Separation}

Suppose that the two industrial production patterns in the same production line are Modes $A$ and $B . X_{A}$ and $X_{B}$ are the original process data sets of Modes $A$ and $B$, where $X_{A}=\left[X_{1}^{A}, \ldots, X_{N}^{A}\right]^{T} \in(N \times J), X_{B}=\left[X_{1}^{B}, \ldots, X_{N}^{B}\right]^{T} \in$ $(N \times J), Y_{A}$ and $Y_{B}$ are their quality data sets, where $Y_{A}=$ $\left[Y_{1}^{A}, \ldots, Y_{N}^{A}\right]^{T} \in(N \times J), Y_{B}=\left[Y_{1}^{B}, \ldots, Y_{N}^{B}\right]^{T} \in(N \times J)$. In addition, $J$ and $N$ are the variable number and sampling points, respectively. The extraction steps of the quality-related common subspace are as follows.

(1) Map the original process data and quality data from the original space to the feature space as $X_{A} \rightarrow$ $\Phi\left(X_{A}\right), X_{B} \rightarrow \Phi\left(X_{B}\right), Y_{A} \rightarrow \Phi\left(Y_{A}\right) Y_{B} \rightarrow \Phi\left(Y_{B}\right)$.

(2) Find the relationship between quality variables and process variables to get the quality-related process data: $\Phi\left(X_{A}\right)+\Phi\left(Y_{A}\right) \rightarrow \widehat{\Phi}\left(X_{A}\right), \Phi\left(X_{B}\right)+\Phi\left(Y_{B}\right) \rightarrow \widehat{\Phi}\left(X_{B}\right)$.

(3) Put $\widehat{\Phi}\left(X_{A}\right)$ and $\widehat{\Phi}\left(X_{B}\right)$ together as the new data sets: $\widehat{\Phi}(X)=\widehat{\Phi}\left(X_{A}\right)+\widehat{\Phi}\left(X_{B}\right)$.

(4) Extract the quality-related common subspace: $\widehat{\Phi}(X) \rightarrow \widehat{\Phi}\left(X^{C}\right)$.

Important symbols and their meanings are listed in the abbreviation section.

In the second step, the interrelation of quality variables and process variables is establishedas follows:

$$
\begin{array}{ll}
\max & w_{A}^{T} \Phi\left(X_{A}\right)^{T} \Phi\left(Y_{A}\right) c_{A} \\
\text { s.t. } & \left\|w_{A}\right\|^{2}=\left\|c_{A}\right\|^{2}=1, \\
\max & w_{B}^{T} \Phi\left(X_{B}\right)^{T} \Phi\left(Y_{B}\right) c_{B} \\
\text { s.t. } & \left\|w_{B}\right\|^{2}=\left\|c_{B}\right\|^{2}=1 .
\end{array}
$$

In the formulas (1), $w_{A}$ and $c_{A}$ are scores of the process variables and the quality variables of Mode $A$, respectively, and $w_{B}$ and $c_{B}$ are scores of the process variables and the quality variables of Mode $B$, respectively. Using the formulas above, we can get quality-related process data $\widehat{\Phi}\left(X_{A}\right)$ and $\widehat{\Phi}\left(X_{B}\right)$.

For every data point of the feature space $\widehat{\Phi}\left(X_{i}\right) \in \widehat{\Phi}(X)$, find the $k$ nearest neighbors in $\chi_{i}=\left\{\widehat{\Phi}\left(X_{i_{j}}\right)\right\}_{j=1}^{k}$. In addition, $\chi_{i}=\left\{\widehat{\Phi}\left(X_{i_{j}}\right)\right\}_{j=1}^{k}$ are centered nonlinear mappings of the input variables.

Project $k$ data points in $\chi_{i}$ into the tangent space at $\widehat{\Phi}\left(X_{i}\right)$, and linearly align the local coordinates into a single global coordinate system in $R^{d}$. Consider

$$
\widehat{\Phi}\left(\mathbf{X}_{i_{j}}^{c}\right) \approx \mathbf{L}_{i} \mathbf{Q}_{i}^{T}\left(\widehat{\Phi}\left(\mathbf{X}_{i_{j}}\right)-\mathbf{C}_{i}\right)+\mathbf{b}_{i}, \quad j=1,2, \ldots, k,
$$

where $\mathbf{t}_{j}^{(i)}=\mathbf{Q}_{i}^{t}\left(\widehat{\Phi}\left(\mathbf{X}_{i_{j}}\right)-\mathbf{C}_{i}\right), j=1,2, \ldots k$ is named a local coordinate of $\widehat{\Phi}\left(\mathbf{X}_{i_{j}}\right), \mathbf{C}_{i}=(1 / k) \sum_{j=1}^{k} \widehat{\Phi}\left(\mathbf{X}_{i_{j}}\right)$ is the mean vector, and $\mathbf{Q}_{i}$ is a tangent space projection matrix. $\mathbf{Q}_{i}$ can be estimated by performing the optimal rank- $d$ approximation of the centered data matrix. $\mathbf{L}_{i} \in \mathbf{R}^{(d \times d)}$ is an affine transformation matrix and $\mathbf{b}_{i} \in \mathbf{R}^{d}$ is a translation vector.

Consider $\mathbf{Q}_{i} \mathbf{L}_{i}^{T}$ in (2) as a transformation matrix; a linear transformation can be obtained as follows:

$$
\widehat{\Phi}\left(\mathbf{X}_{i_{j}}^{C}\right) \approx \mathbf{W}_{i}^{T}\left(\widehat{\Phi}\left(\mathbf{X}_{i_{j}}\right)-\mathbf{C}_{i}\right)+\mathbf{b}_{i}, \quad j=1,2, \ldots, k
$$

where $\mathbf{W}_{i}=\mathbf{Q}_{i} \mathbf{L}_{i}^{T},\|\mathbf{W}\|_{2}^{2}=1$.

Solve $\mathbf{W}_{i}$ and $\mathbf{b}_{i}$ under the least-square regression. Obviously, the result is expected that each $\widehat{\Phi}\left(\mathbf{X}_{i_{j}}\right)$ can be directly mapped as $\widehat{\Phi}\left(\mathbf{X}_{i_{j}}^{C}\right)$. Consider

$$
\widehat{\Phi}\left(\mathbf{X}_{i_{j}}^{C}\right) \approx g_{i}\left(\widehat{\Phi}\left(\mathbf{X}_{i_{j}}\right)\right)
$$

where $g_{i}(x)$ is a function to make the measurement data approximate the common subspace data point $\widehat{\Phi}\left(\mathbf{X}_{i_{j}}^{C}\right)$.

The least-square regression problem is given as follows:

$$
\min \sum_{j=1}^{k}\left\|\widehat{\Phi}\left(\mathbf{X}_{i_{j}}^{C}\right)-g\left(\widehat{\Phi}\left(\mathbf{X}_{i_{j}}\right)\right)\right\|_{2}^{2}+\lambda\left\|\mathbf{W}_{i}\right\|_{2}^{2},
$$

where $\lambda$ is a positive parameter. 
According to (5), the object function $G\left(\mathbf{W}_{i}, \mathbf{b}_{i}\right)$ is as follows:

$$
\begin{gathered}
G\left(\mathbf{W}_{i}, \mathbf{b}_{i}\right) \\
=\min \sum_{j=1}^{k}\left\|\mathbf{W}_{i}^{T}\left(\widehat{\Phi}\left(\mathbf{X}_{i_{j}}\right)-\mathbf{C}_{i}\right)+\mathbf{b}_{i}-g\left(\widehat{\Phi}\left(\mathbf{X}_{i_{j}}\right)\right)\right\|_{2}^{2} \\
+\lambda\left\|\mathbf{W}_{i}\right\|_{2}^{2} .
\end{gathered}
$$

Let the replacing matrix $\widehat{\Phi}\left(\mathbf{X}_{i}^{C}\right)=\left[g\left(\widehat{\Phi}\left(\mathbf{X}_{i_{1}}\right)\right)-\right.$ $\left.\mathbf{C}_{i}, g\left(\widehat{\Phi}\left(\mathbf{X}_{i_{2}}\right)\right)-\mathbf{C}_{i}, \ldots, g\left(\widehat{\Phi}\left(\mathbf{X}_{i_{k}}\right)\right)-\mathbf{C}_{i}\right]$ be the centralized data matrix and let $\widehat{\Phi}\left(\mathbf{X}_{i}^{C}\right)=\left[\widehat{\Phi}\left(\mathbf{X}_{i_{1}}^{C}\right), \widehat{\Phi}\left(\mathbf{X}_{i_{2}}^{C}\right), \ldots, \widehat{\Phi}\left(\mathbf{X}_{i_{k}}^{C}\right)\right]$ collect the $k$ data points.

The optimization $\mathbf{W}_{i}$ and $\mathbf{b}_{i}$ should meet the following conditions:

$$
\begin{aligned}
& \frac{\partial G\left(\mathbf{W}_{i}, \mathbf{b}_{i}\right)}{\partial \mathbf{W}_{i}}=\mathbf{0}, \\
& \frac{\partial G\left(\mathbf{W}_{i}, \mathbf{b}_{i}\right)}{\partial \mathbf{b}_{i}}=\mathbf{0} .
\end{aligned}
$$

Then

$$
\begin{aligned}
\frac{\partial G\left(\mathbf{W}_{i}, \mathbf{b}_{i}\right)}{\partial \mathbf{W}_{i}}= & \mathbf{0} \\
\Longrightarrow & 2 \lambda \mathbf{W}_{i}+2 \widehat{\Phi}\left(\mathbf{X}_{i}^{C}\right) \\
& \times\left(\widehat{\Phi}\left(\mathbf{X}_{i}^{C}\right)^{T} \mathbf{W}_{i}+\mathbf{e}_{k} \mathbf{b}_{i}^{T}-\widehat{\Phi}\left(\mathbf{X}_{i}^{C}\right)^{T}\right)=\mathbf{0} \\
\Longrightarrow & \left(\widehat{\Phi}\left(\mathbf{X}_{i}^{C}\right) \widehat{\Phi}\left(\mathbf{X}_{i}^{C}\right)^{T}+\lambda \mathbf{I}_{m}\right) \mathbf{W}_{i} \\
= & \widehat{\Phi}\left(\mathbf{X}_{i}^{C}\right)\left(\widehat{\Phi}\left(\mathbf{X}_{i}^{C}\right)^{T}-\mathbf{e}_{k} \mathbf{b}_{i}^{T}\right) \\
\Longrightarrow & \mathbf{W}_{i}=\left(\widehat{\Phi}\left(\mathbf{X}_{i}^{C}\right) \widehat{\Phi}\left(\mathbf{X}_{i}^{C}\right)^{T}+\lambda \mathbf{I}_{m}\right)^{-1} \\
& \times \widehat{\Phi}\left(\mathbf{X}_{i}^{C}\right) \widehat{\Phi}\left(\mathbf{X}_{i}^{C}\right)^{T}, \\
\frac{\partial G\left(\mathbf{W}_{i}, \mathbf{b}_{i}\right)}{\partial \mathbf{b}_{i}}= & \mathbf{0} \\
\Longrightarrow & 2\left(\mathbf{W}_{i}^{T} \widehat{\Phi}\left(\mathbf{X}_{i}^{C}\right) \mathbf{e}_{k}+\mathbf{b}_{i} \mathbf{e}_{k}^{T} \mathbf{e}_{k}-\widehat{\Phi}\left(\mathbf{X}_{i}^{C}\right) \mathbf{e}_{k}\right) \\
= & \mathbf{0} \\
= & \frac{1}{k} \widehat{\Phi}\left(\mathbf{X}_{i}=\widehat{\Phi}\left(\mathbf{X}_{i}^{C}\right) \mathbf{e}_{k}-\mathbf{W}_{i}^{T} \widehat{\Phi}\left(\mathbf{X}_{i}^{C}\right) \mathbf{e}_{k}\right. \\
= & \mathbf{b}_{i}=\frac{1}{k}\left(\widehat{\Phi}\left(\mathbf{X}_{i}^{C}\right) \mathbf{e}_{k}-\mathbf{W}_{i}^{T} \widehat{\Phi}\left(\mathbf{X}_{i}^{C}\right) \mathbf{e}_{k}\right) \\
&
\end{aligned}
$$

Here, the last equality holds because $\widehat{\Phi}\left(\mathbf{X}_{i}^{C}\right) \mathbf{e}_{k}=\mathbf{0}$. Thus, the optimal $\mathbf{W}_{i}$ and $\mathbf{b}_{i}$ can be obtained as follows:

$$
\begin{aligned}
\mathbf{W}_{i} & =\left(\widehat{\Phi}\left(\mathbf{X}_{i}^{C}\right) \widehat{\Phi}\left(\mathbf{X}_{i}^{C}\right)^{T}+\lambda \mathbf{I}_{m}\right)^{-1} \widehat{\Phi}\left(\mathbf{X}_{i}^{C}\right) \widehat{\Phi}\left(\mathbf{X}_{i}^{C}\right)^{T}, \\
\mathbf{b}_{i} & =\frac{1}{k} \widehat{\Phi}\left(\mathbf{X}_{i}^{C}\right) \mathbf{e}_{k} .
\end{aligned}
$$

After the optimal $\mathbf{W}_{i}$ and $\mathbf{b}_{i}$ are estimated, the sum of squared errors can be evaluated as follows:

$$
\varepsilon_{i}=\sum_{j=i}^{k}\left\|\widehat{\Phi}\left(\mathbf{X}_{i_{j}}^{C}\right)-\left(\mathbf{W}_{i}^{T}\left(\widehat{\Phi}\left(\mathbf{X}_{i_{j}}\right)-\mathbf{C}_{i}\right)+\mathbf{b}_{i}\right)\right\| .
$$

So we can get (11) from (9) as follows:

$$
\begin{aligned}
& \varepsilon_{i}=\sum_{j=i}^{k}\left\|\widehat{\Phi}\left(\mathbf{X}_{i_{j}}^{C}\right)-\left(\mathbf{W}_{i}^{T}\left(\widehat{\Phi}\left(\mathbf{X}_{i_{j}}\right)-\mathbf{C}_{i}\right)+\mathbf{b}_{i}\right)\right\|_{2}^{2} \\
&=\operatorname{tr}(\left(\widehat{\Phi}\left(\mathbf{X}_{i}^{C}\right)-\mathbf{W}_{i}^{T} \widehat{\Phi}\left(\mathbf{X}_{i}^{C}\right)-\mathbf{b}_{i} \mathbf{e}_{k}^{T}\right) \\
&\left.\times\left(\widehat{\Phi}\left(\mathbf{X}_{i}^{C}\right)-\mathbf{W}_{i}^{T} \widehat{\Phi}\left(\mathbf{X}_{i}^{C}\right)-\mathbf{b}_{i} \mathbf{e}_{k}^{T}\right)^{T}\right) .
\end{aligned}
$$

Let $\widehat{\Phi}\left(\mathbf{X}_{i}^{C}\right)=\widehat{\Phi}\left(\mathbf{X}_{i}^{C}\right) \mathbf{L}$ and symmetric matrix $\mathbf{L}=\mathbf{L}^{T}=\mathbf{L}^{2}$. Then, simplify the expression as follows:

$$
\begin{aligned}
\widehat{\Phi}\left(\mathbf{X}_{i}^{C}\right)-\mathbf{W}_{i}^{T} \widehat{\Phi}\left(\mathbf{X}_{i}^{C}\right)-\mathbf{b}_{i} \mathbf{e}_{k}^{T} \\
=\widehat{\Phi}\left(\mathbf{X}_{i}^{C}\right) \mathbf{L}\left(\mathbf{I}_{k}-\widehat{\Phi}\left(\mathbf{X}_{i}^{C}\right)^{T}\right. \\
\times\left(\widehat{\Phi}\left(\mathbf{X}_{i}^{C}\right) \widehat{\Phi}\left(\mathbf{X}_{i}^{C}\right)^{T}+\lambda \mathbf{I}_{m}\right)^{-1} \\
\left.\times \widehat{\Phi}\left(\mathbf{X}_{i}^{C}\right)\right) .
\end{aligned}
$$

Let

$$
\begin{aligned}
\mathbf{G}_{i}= & \mathbf{I}_{k}-\widehat{\Phi}\left(\mathbf{X}_{i}^{C}\right)^{T}\left(\widehat{\Phi}\left(\mathbf{X}_{i}^{C}\right) \widehat{\Phi}\left(\mathbf{X}_{i}^{C} s\right)^{T}+\lambda \mathbf{I}_{m}\right)^{(-1)} \\
& \times \widehat{\Phi}\left(\mathbf{X}_{i}^{C}\right),
\end{aligned}
$$

where both $\mathbf{I}_{m}$ and $\mathbf{I}_{k}$ are identity matrices and $\mathbf{G}_{i}$ is symmetrical. Thus,

$$
\varepsilon_{i}=\operatorname{tr}\left(\widehat{\Phi}\left(\mathbf{X}_{i}^{C}\right) \mathbf{L G}_{i} \mathbf{G}_{i} \mathbf{L} \widehat{\Phi}\left(\mathbf{X}_{i}^{C}\right)^{T}\right) .
$$

When the sum of the squared errors in each neighborhood $\chi_{i}$ is determined, an objective function can be constructed to express the global embedding as follows:

$$
\mathbf{E}\left(\mathbf{X}_{i}^{C}\right)=\sum_{i=1}^{n} \operatorname{tr}\left(\widehat{\Phi}\left(\mathbf{X}_{i}^{C}\right) \mathbf{L} \mathbf{G}_{i} \mathbf{G}_{i} \mathbf{L} \widehat{\Phi}\left(\mathbf{X}_{i}^{C}\right)^{T}\right) .
$$

Solve the following problem, and obtain the embedding:

$$
\begin{array}{ll}
\min & \operatorname{tr}\left(\widehat{\Phi}\left(\mathbf{X}_{i}^{C}\right) \mathbf{L G}_{i} \mathbf{G}_{i} \mathbf{L} \widehat{\Phi}\left(\mathbf{X}_{i}^{C}\right)^{T}\right) \\
\text { s.t. } & \widehat{\Phi}\left(\mathbf{X}_{i}^{C}\right)^{T} \widehat{\Phi}\left(\mathbf{X}_{i}^{C}\right)=\mathbf{I}_{d} .
\end{array}
$$


From (16), $\widehat{\Phi}\left(\mathbf{X}_{i}^{C}\right)$ can be gotten corresponding once the minimum trace was determined. In consequence we can obtain the quality-related common subspace $\widehat{\Phi}\left(\mathbf{X}^{C}\right)=$ $\left[\widehat{\Phi}\left(\mathbf{X}_{1}^{C}\right), \widehat{\Phi}\left(\mathbf{X}_{2}^{C}\right), \ldots, \widehat{\Phi}\left(\mathbf{X}_{n}^{C}\right)\right]$.

After obtaining the quality-related common subspace, we can decompose the quality-related process data of Mode $A$ and Mode $B$ into two different parts: the quality-related common subspace and two quality-related specific subspaces are independent to each other. One has

$$
\begin{aligned}
& \widehat{\Phi}\left(\mathbf{X}_{A}\right)=\widehat{\Phi}\left(\mathbf{X}^{C}\right)+\widehat{\Phi}\left(\mathbf{X}_{A}^{S}\right), \\
& \widehat{\Phi}\left(\mathbf{X}_{B}\right)=\widehat{\Phi}\left(\mathbf{X}^{C}\right)+\widehat{\Phi}\left(\mathbf{X}_{B}^{S}\right) .
\end{aligned}
$$

Next, we built the principal component models of the common subspace and two specific subspaces as follows:

$$
\begin{aligned}
\widehat{\Phi}\left(\mathbf{X}^{C}\right) & =\widetilde{\widehat{\Phi}}\left(\mathbf{X}^{C}\right)+\widehat{\mathbf{E}}, \\
\widetilde{\Phi}\left(\mathbf{X}^{C}\right) & =\widehat{\mathbf{T}}^{C} \widehat{\mathbf{P}}_{g}^{T}, \\
\widehat{\mathbf{E}} & =\widehat{\mathbf{T}}_{e}^{C} \widehat{\mathbf{P}}_{e}^{T}, \\
\widehat{\Phi}\left(\mathbf{X}_{A}^{S}\right) & =\widetilde{\widehat{\Phi}}\left(\mathbf{X}_{A}^{S}\right)+\widehat{\mathbf{E}}_{A}^{S}, \\
\widetilde{\Phi}\left(\mathbf{X}_{A}^{S}\right) & =\widehat{\mathbf{T}}_{A}^{S} \widehat{\mathbf{P}}_{A}^{T}, \\
\widehat{\mathbf{E}}_{A}^{S} & =\widehat{\mathbf{T}}_{e, A}^{S} \widehat{\mathbf{P}}_{e, A}^{S}, \\
\widehat{\Phi}\left(\mathbf{X}_{B}^{S}\right) & =\widetilde{\widehat{\Phi}}_{\left(\mathbf{X}_{B}^{S}\right)+\widehat{\mathbf{E}}_{B}^{S},} \\
\widetilde{\Phi}\left(\mathbf{X}_{B}^{S}\right) & =\widehat{\mathbf{T}}_{B}^{S} \widehat{\mathbf{P}}_{B}^{T}, \\
\widehat{\mathbf{E}}_{B}^{S} & =\widehat{\mathbf{T}}_{e, B}^{S} \widehat{\mathbf{P}}_{e, B}^{S},
\end{aligned}
$$

where $\widehat{\mathbf{P}}_{g}(J \times R)$ is the common loading and $R$ is the PC number. $\widehat{\mathbf{P}}_{A}\left(J \times R_{A}\right)$ and $\mathbf{T}_{S, B}^{2}=\left(\mathbf{t}_{\text {new }, B}^{S}\right)^{T}\left(\Lambda_{B}^{S}\right)^{-1} \mathbf{t}_{\text {new, } B}^{S}$ are the loadings of two quality-related specific subspaces; $R_{A}$ and $R_{B}$ are the retained PC numbers in the two qualityrelated specific subspaces. $\widehat{\mathbf{E}}_{A}^{S}$ and $\widehat{\mathbf{E}}_{B}^{S}$ are the specific residual matrixes.

\section{Monitoring of Multimode Processes}

To verify the validity of the method, we can monitor the Hotelling- $T^{2}$ and SPE, respectively, in quality-related common subspace, quality-related specific subspaces, and residuals spaces.

Assume $\mathbf{x}_{\text {new }}(J \times 1)$ is the new observation vector, which has been normalized by the mean and variance in advance. To calculate the common score, we project it onto the unified common subspace. The common score and Hotelling- $T^{2}$ statistic are calculated as follows:

$$
\begin{aligned}
\widehat{\mathbf{t}}_{\text {new }}^{C} & =\widehat{\mathbf{P}}_{g}^{T} \Phi\left(\mathbf{x}_{\text {new }}\right), \\
\widehat{\mathbf{T}}_{c}^{2} & =\left(\widehat{\mathbf{t}}_{\text {new }}^{C}\right)^{T}\left(\Lambda^{C}\right)^{-1}\left(\widehat{\mathbf{t}}_{\text {new }}^{C}\right),
\end{aligned}
$$

where $\Lambda^{C}$ is the covariance matrix of the common subspace, which is also connected with the retained PCs.

Use the following formulas to calculate the corresponding specific score and the Hotelling- $T^{2}$ statistic in quality-related specific subspaces:

$$
\begin{aligned}
\widehat{\mathbf{t}}_{\text {new }, A}^{S} & =\widehat{\mathbf{P}}_{A}^{T} \mathbf{x}_{\text {new }}, \\
\widehat{\mathbf{t}}_{\text {new }, B}^{S} & =\widehat{\mathbf{P}}_{B}^{T} \mathbf{x}_{\text {new }}, \\
\widehat{\mathbf{T}}_{S, A}^{2} & =\left(\widehat{\mathbf{t}}_{\text {new }, A}^{S}\right)^{T}\left(\Lambda_{A}^{S}\right)^{-1} \widehat{\mathbf{t}}_{\text {new }, A}^{S}, \\
\widehat{\mathbf{T}}_{S, B}^{2} & =\left(\widehat{\mathbf{t}}_{\text {new }, B}^{S}\right)^{T}\left(\Lambda_{B}^{S}\right)^{-1} \widehat{\mathbf{t}}_{\text {new }, B}^{S}, \\
\widehat{\mathbf{x}}_{\text {new }}^{C} & =\widehat{\mathbf{P}}_{g} \widehat{\mathbf{t}}_{\text {new }}^{C}, \\
\widehat{\mathbf{x}}_{\text {new }}^{S} & =\mathbf{x}_{\text {new }}-\widehat{\mathbf{x}}_{\text {new }}^{C}, \\
\widetilde{\mathbf{x}}_{\text {new }, m}^{S} & =\widehat{\mathbf{P}}_{m} \widehat{\mathbf{t}}_{\text {new }, m}^{S}, \quad m=A, B, \\
\widehat{\mathbf{e}}_{\text {new }, m}^{S} & =\widehat{\mathbf{x}}_{\text {new }}^{S}-\widetilde{\mathbf{x}}_{\text {new }, m}^{S}, \\
\mathrm{SPE}_{\text {new }, m} & =\left(\widehat{\mathbf{e}}_{\text {new }, m}^{S}\right)^{T} \widehat{\mathbf{e}}_{\text {new }, m}^{S} .
\end{aligned}
$$

Use the Hotelling- $T^{2}$ to detect the faults of the common features. If the result shows there are no faults in the common features, the specific Hotelling- $T^{2}$ can reflect the faults in the specific features. Simultaneously, the value of SPE can be used to detect the process faults in residual space.

\section{Experimental Results}

We add the correlation between process variables and quality variables in model based on the traditional subspace separation method and then apply the new multimode monitoring method to the process of the electrical fused magnesium furnace (EFMF). Simulation experiment verifies the method above is feasible. Firstly, define the normal process and quality data of Mode $A$ and Mode $B$ as, $\mathbf{X}_{A}, \mathbf{X}_{B}, \mathbf{Y}_{A}$, and $\mathbf{Y}_{B}$, respectively. There are seven crucial variables in each column of the data sets, including the value of three-phase current, the value of three-phase voltage, and the relative position of the electrode. The furnace temperature is the only quality data. A normal batch run which belongs to Mode $A$ contains 400 sampling points. It is used to verify the feasibility of the above-proposed method. The Hotelling- $T^{2}$ statistics values, which are dependent on the confidence region of the common subspace, have been shown in Figure 1(a). In the case of the different specific subspaces, as is shown in Figures 1(b) and 1(c), the Hotelling- $T^{2}$ statistics values depend on the confidence region in Mode $A$ only. We will know the operating mode of the EFMF from Figure 1. Both Hotelling$T^{2}$ and SPE statistical variables of Mode $A$ can show there are no faults. In the specific space of Mode $B, \mathrm{SPE}$ shows normal, while Hotelling- $T^{2}$ detects the fault. From the test results we have reason to think that the test data are normal data belonging to Mode $A$ rather than Mode $B$. 


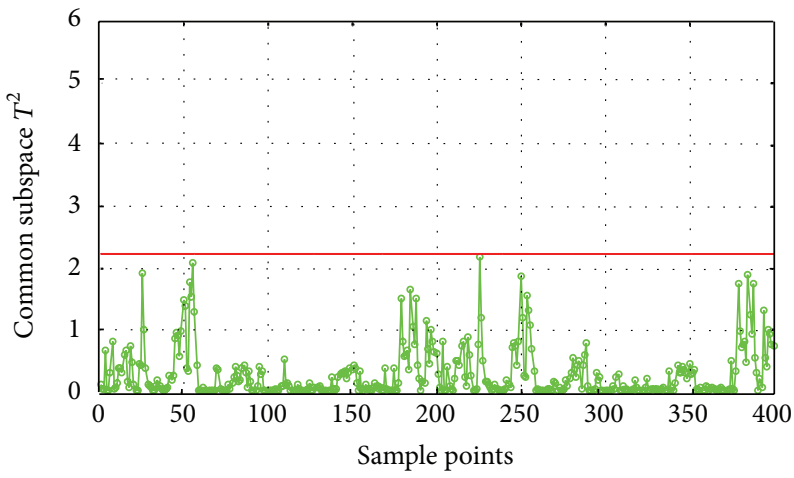

(a)
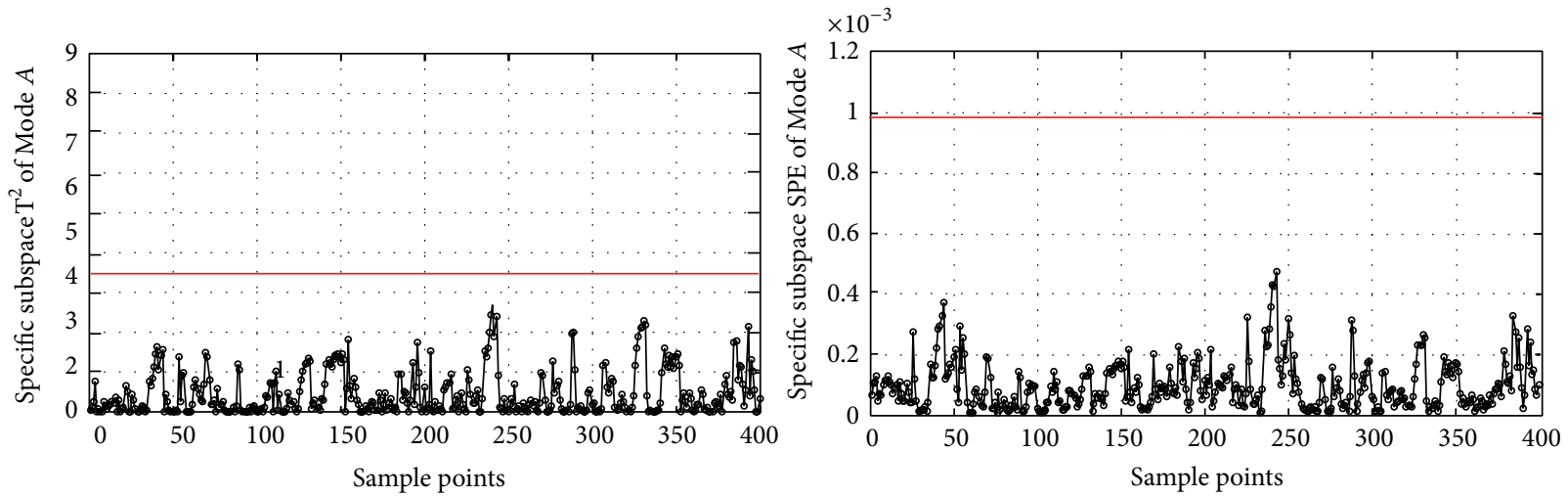

(b)
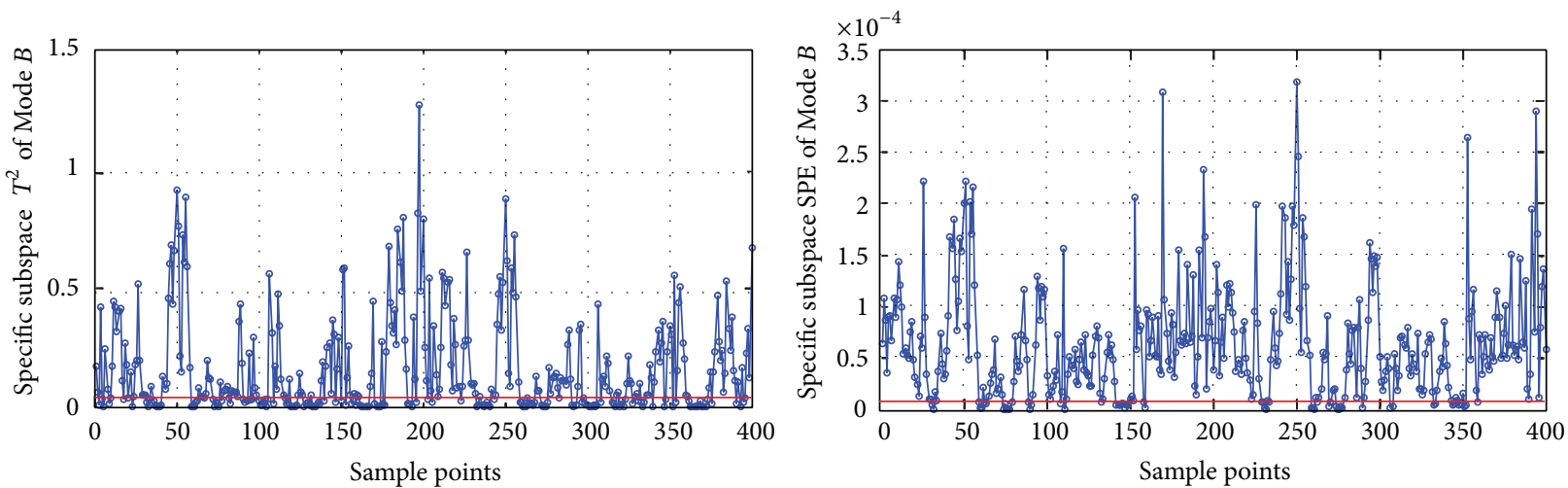

(c)

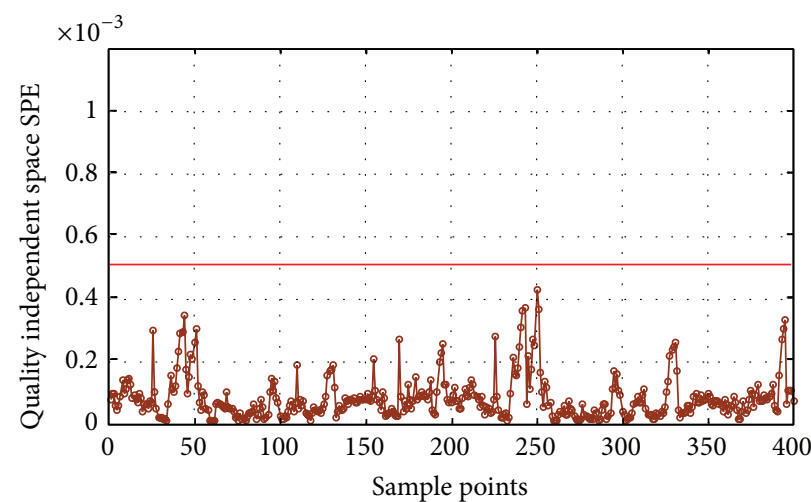

(d)

FIGURE 1: The proposed method monitoring results of the two different modes for (a) quality-related common part $T^{2}$ statistic, (b) qualityrelated specific part $T^{2}$ and SPE statistic of Mode $A$, and (c) quality-related specific part $T^{2}$ and SPE statistic of Mode $B$. 


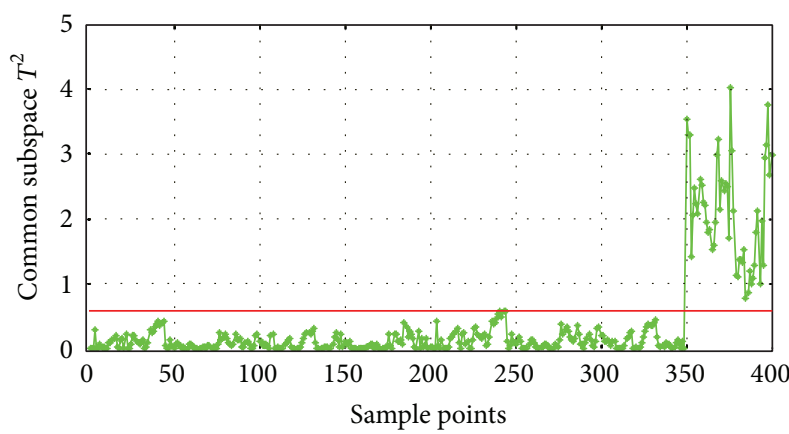

(a)

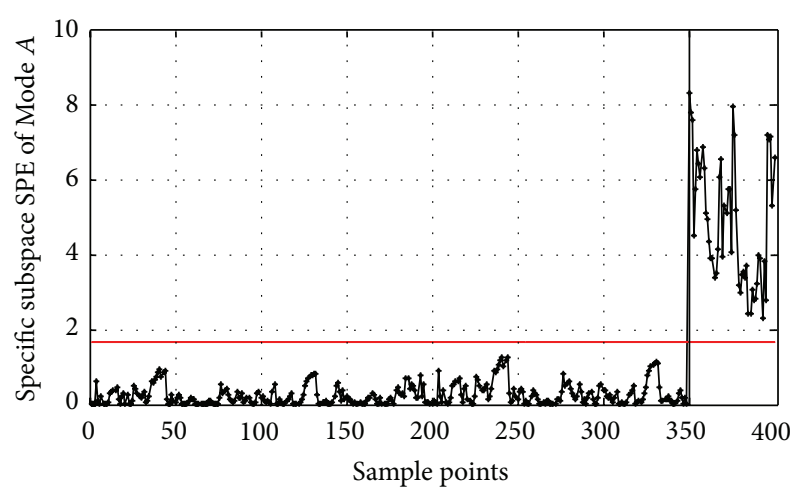

(c)

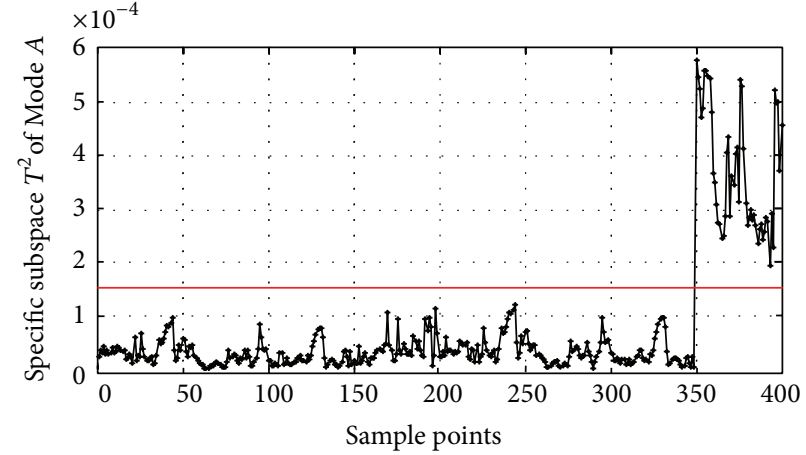

(b)

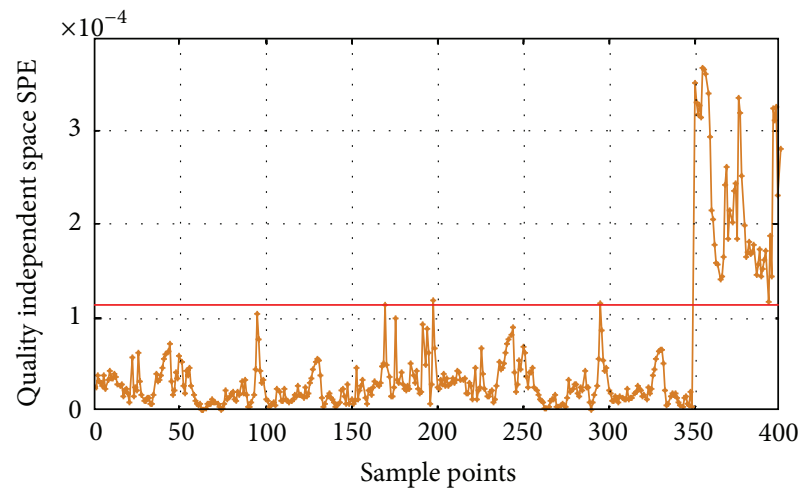

(d)

Figure 2: Monitoring results by Mode $A$ for (a) quality-related common part $T^{2}$ statistic, (b) quality-related specific part $T^{2}$ statistic, (c) quality-related residual statistic, and (d) quality-unrelated residual statistic.

Next, we use the above-proposed method to monitor an abnormal batch run which belongs to Mode A. Previously, we introduced process faults from the 350th sample point. As shown in Figure 2, the proposed method can detect the fault effectively in quality-related common space, specific space, and residual space.

\section{Conclusion}

In this paper, the proposed approach, monitoring for multimode processes based on quality-related common subspace separation, is feasible. It makes the correlation between the quality-related common subspace and each mode data set and also reduces the complexity and fault rate of the monitoring program. Utilize the EFMF process as a backdrop; simulation experiment verifies the method above is effective. In the experiment we can see how the proposed method performs, which shows the desirable improvement in multimode processes. As application method, it should be applied in many similar multimode processes to help understanding and improving the monitoring model.

\section{Abbreviations}

$X_{A}$ : Raw normal process data of Mode $A$

$X_{B}$ : Raw normal process data of Mode $B$
$Y_{A}: \quad$ Raw quality data of Mode $A$

$Y_{B}$ : $\quad$ Raw quality data of Mode $B$

$\Phi\left(X_{A}\right)$ : Process data mapped into high-dimensional space of Mode $A$

$\Phi\left(X_{B}\right)$ : Process data mapped into high-dimensional space of Mode $B$

$\Phi\left(Y_{A}\right)$ : Quality data mapped into high-dimensional space of Mode $A$

$\Phi\left(Y_{B}\right)$ : Quality data mapped into high-dimensional space of Mode $B$

$\widehat{\Phi}\left(X_{A}\right)$ : Quality-related process data mapped into high-dimensional space of Mode $A$

$\widehat{\Phi}\left(X_{B}\right)$ : Quality-related process data mapped into high-dimensional space of Mode $B$

$\widehat{\Phi}\left(X_{i}^{C}\right)$ : Component of quality common subspace

$\widehat{\Phi}\left(X^{C}\right)$ : Quality-related common subspace

$\widehat{\Phi}\left(X_{A}^{S}\right)$ : Quality-related specific subspace of Mode $A$

$\widehat{\Phi}\left(X_{B}^{S}\right)$ : Quality-related specific subspace of Mode $B$.

\section{Conflict of Interests}

The authors declare that there is no conflict of interests regarding the publication of this paper. 


\section{References}

[1] Z. Gao, X. Dai, T. Breikin, and H. Wang, "Novel parameter identification by using a high-gain observer with application to a gas turbine engine," IEEE Transactions on Industrial Informatics, vol. 4, no. 4, pp. 271-279, 2008.

[2] Z. W. Gao, T. Breikin, and H. Wang, "High-gain estimator and fault-tolerant design with application to a gas turbine dynamic system," IEEE Transactions on Control Systems Technology, vol. 15, no. 4, pp. 740-753, 2007.

[3] M. H. Kim, S. Lee, and K. C. Lee, "Kalman predictive redundancy system for fault tolerance of safety-critical systems," IEEE Transactions on Industrial Informatics, vol. 6, no. 1, pp. 46-53, 2010.

[4] G. Gaderer, P. Loschmidt, and T. Sauter, "Improving fault tolerance in high-precision clock synchronization," IEEE Transactions on Industrial Informatics, vol. 6, no. 2, pp. 206-215, 2010.

[5] T. J. Harris, C. T. Seppala, and L. D. Desborough, "A review of performance monitoring and assessment techniques for univariate and multivariate control systems," Journal of Process Control, vol. 9, no. 1, pp. 1-17, 1999.

[6] V. Venkatasubramanian, R. Rengaswamy, K. Yin, and S. N. Kavuri, "A review of process fault detection and diagnosis part I: quantitative model-based methods," Computers and Chemical Engineering, vol. 27, no. 3, pp. 293-311, 2003.

[7] P. Miller, R. E. Swanson, and C. E. Heckler, "Contribution plots: a missing link in multivariate quality control," Applied Mathematics and Computer Science, vol. 8, no. 4, pp. 775-792, 1998.

[8] J. Chen and K. Liu, "On-line batch process monitoring using dynamic PCA and dynamic PLS models," Chemical Engineering Science, vol. 57, no. 1, pp. 63-75, 2002.

[9] M. Misra, H. H. Yue, S. J. Qin, and C. Ling, "Multivariate process monitoring and fault diagnosis by multi-scale PCA," Computers and Chemical Engineering, vol. 26, no. 9, pp. 1281-1293, 2002.

[10] S. W. Choi and I. Lee, "Multiblock PLS-based localized process diagnosis," Journal of Process Control, vol. 15, no. 3, pp. 295-306, 2005.

[11] K. X. Peng, K. Zhang, and G. Li, “Total PLS based contribution plots for fault diagnosis," Control Engineering Practice, vol. 21, no. 4, pp. 360-369, 2012.

[12] R. Boqué and A. K. Smilde, "Monitoring and diagnosing batch processes with multiway covariates regression models," AIChE Journal, vol. 45, no. 7, pp. 1504-1520, 1999.

[13] Y. W. Zhang, L. J. Zhang, and H. L. Zhang, "Fault detection for industrial processes," Mathematical Problems in Engineering, vol. 2012, Article ID 757828, 18 pages, 2012.

[14] J. Lee, C. Yoo, and I. Lee, "Statistical process monitoring with independent component analysis," Journal of Process Control, vol. 14, no. 5, pp. 467-485, 2004.

[15] B. Dong, L. Zhang, Y. Wu, J. Feng, and T. Chai, "The fuzzy control research on electrodes of electrical-fused magnesia furnace," in Proceedings of the Chinese Control and Decision Conference (CCDC'08), pp. 216-220, July 2008.

[16] Y. W. Zhang, H. Zhou, S. J. Qin, and T. Chai, "Decentralized fault diagnosis of large-scale processes using multiblock kernel partial least squares," IEEE Transactions on Industrial Informatics, vol. 6, no. 1, pp. 3-10, 2010.

[17] S. Reinikainen and A. Höskuldsson, "Multivariate statistical analysis of a multi-step industrial processes," Analytica Chimica Acta, vol. 595, no. 1-2, pp. 248-256, 2007.
[18] H. H. Ma, Y. Hu, and H. B. Shi, "A novel local neighborhood standardization strategy and its application in fault detection of multimode processes," Chemometrics and Intelligent Laboratory Systems, vol. 118, pp. 287-300, 2012.

[19] Y. Yang and F. Gao, "Adaptive control of the filling velocity of thermoplastics injection molding," Control Engineering Practice, vol. 8, no. 11, pp. 1285-1296, 2000.

[20] J. Yu, "Localized Fisher discriminant analysis based complex chemical process monitoring," AIChE Journal, vol. 57, no. 7, pp. 1817-1828, 2011.

[21] F. L. Wang, S. Tan, J. Peng, and Y. Chang, "Process monitoring based on mode identification for multi-mode process with transitions," Chemometrics and Intelligent Laboratory Systems, vol. 110, no. 1, pp. 144-155, 2012. 


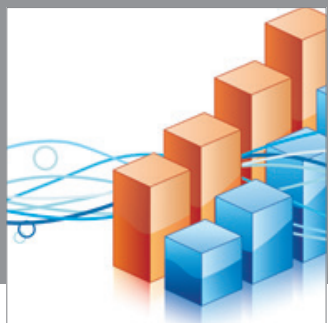

Advances in

Operations Research

mansans

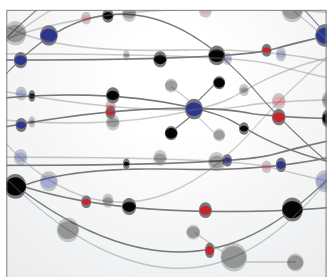

The Scientific World Journal
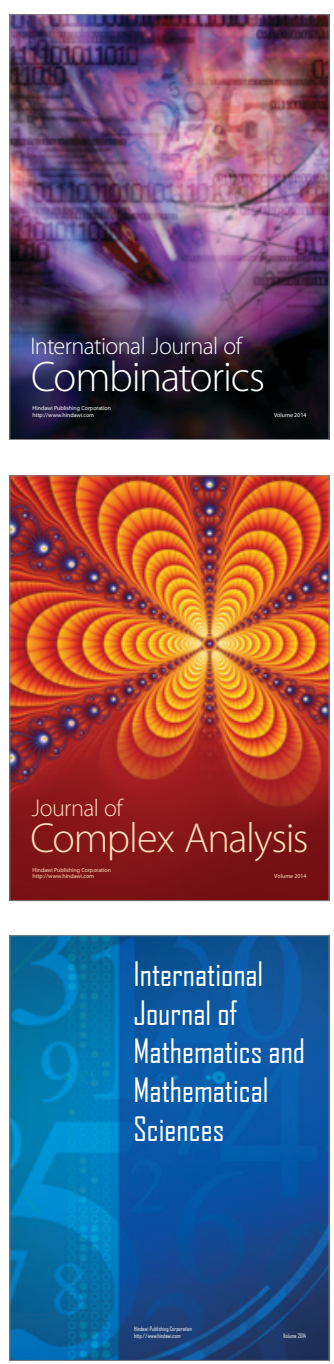
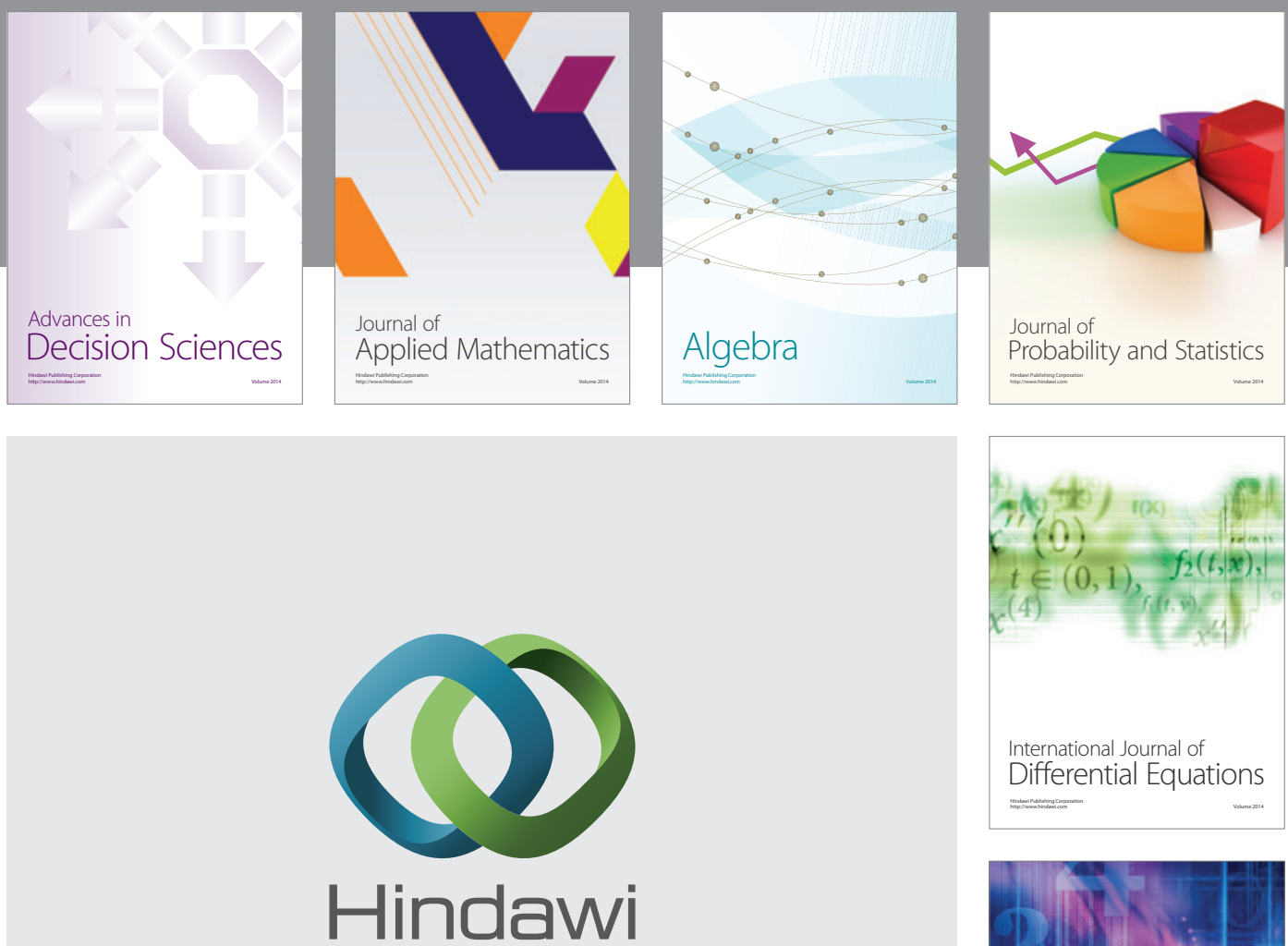

Submit your manuscripts at http://www.hindawi.com
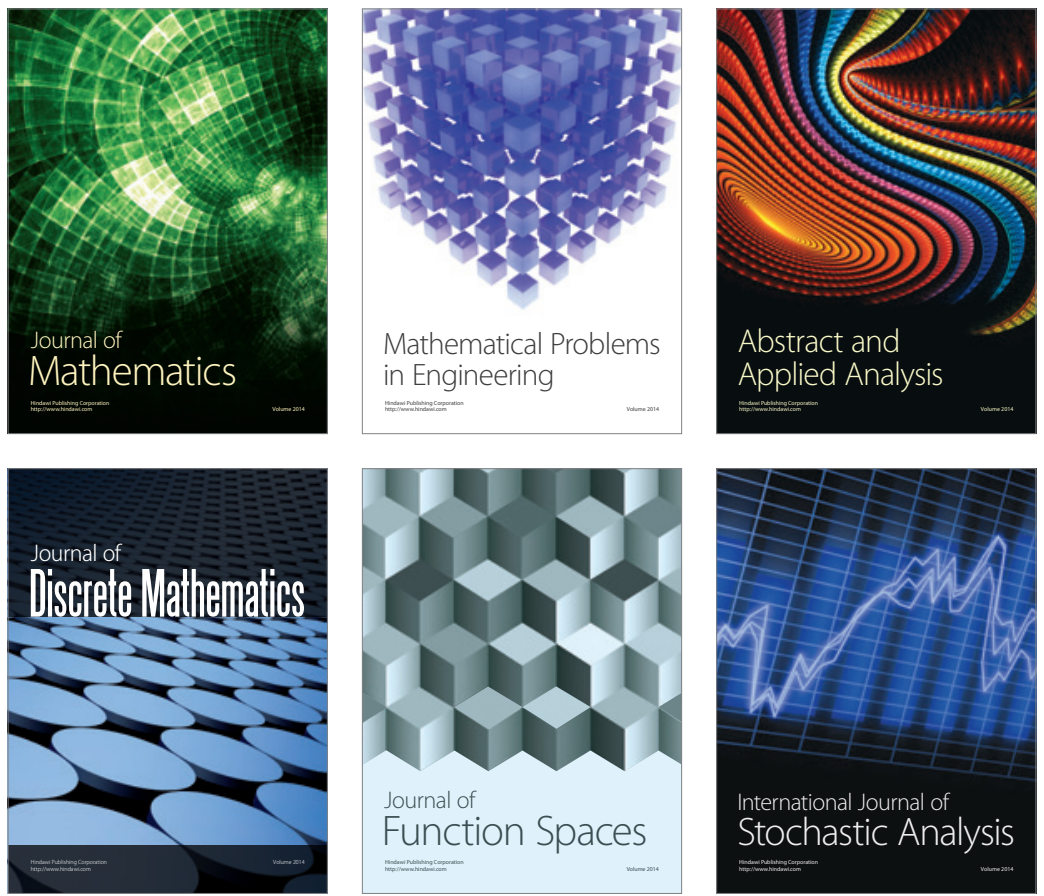

Journal of

Function Spaces

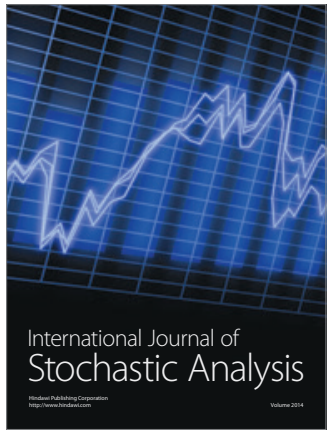

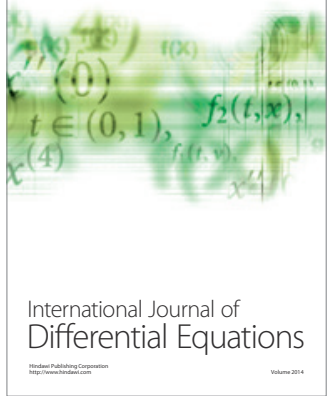
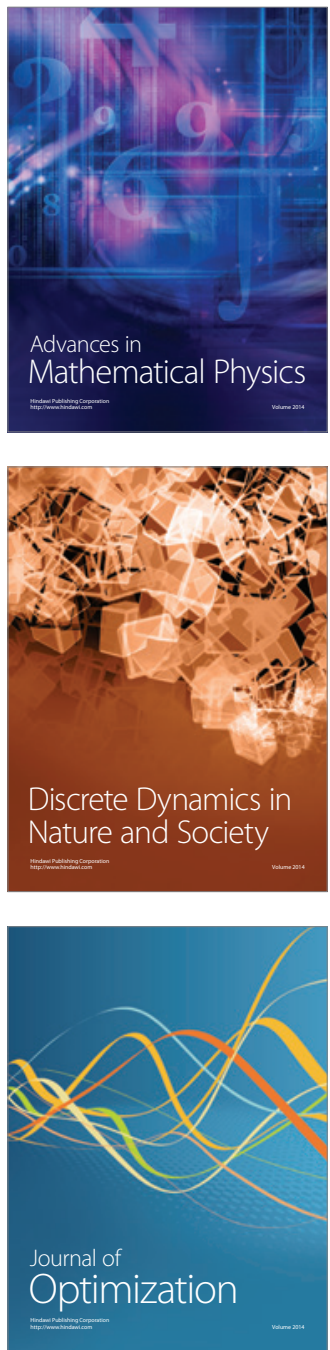